\title{
ÁCIDOS GRASOS OMEGA-3 (EPA Y DHA) Y SU APLICACIÓN EN DIVERSAS SITUACIONES CLÍNICAS
}

\section{OMEGA-3 FATTY ACIDS (EPA AND DHA) AND ITS APPLICATION IN DIVERSE CLINICAL SITUATIONS}

\author{
Rodrigo Valenzuela B. (1), Gladys Tapia O. (2), \\ Marcela González E. (1), Alfonso Valenzuela B. (3)
}

(1) Escuela de Nutrición y Dietética.

Facultad de Medicina. Universidad de Chile. Santiago, Chile.

(2) Programa de Farmacología Molecular y Clínica. ICBM.

Facultad de Medicina. Universidad de Chile. Santiago, Chile.

(3) Centro de Lípidos. Instituto de Nutrición y Tecnología de los Alimentos (INTA).

Universidad de Chile y Facultad de Medicina, Universidad de los Andes. Santiago, Chile.

\begin{abstract}
Omega-3 long-chain polyunsaturated fatty acids ( $\omega$-3 LCPUFA) eicosapentaenoic acid (EPA) and docosahexaenoic acid (DHA) are found in significant amounts in fatty fish (tuna fish, mackerel and salmon) and especially in the oil obtained from these species, which is actually utilized as a nutritional supplement (nutraceutical). After ingestion, both EPA and DHA are rapidly incorporated into cellular membrane phospholipids where they can be released by lipooxygenases and cyclooxygenases enzymes and transformed in powerful bioactive products wich have cytoprotective and especially anti-inflammatory activities. Clinical and epidemiological evidence have firmly established that consumption of EPA and DHA may contribute to the prevention and / or treatment of a number of diseases, especially those where inflammation plays a remarkable role in its development. EPA and DHA exhibit potent anti-inflammatory properties, either via the generation of anti-inflammatory products, such as the resolvins, or by blocking inflammatory agents. In the present paper we review possible clinical applications of EPA and DHA in pathologies, such as cardiovascular and neurodegenerative diseases, cancer, inflammatory bowel disease, rheumatoid arthritis and ischemia - reperfusion injury. The evidence suggests that $\omega-3$ LCPUFA may have promising applications in the prevention and / or treatment of different clinical and nutritional pathologies. Key words: $\omega-3$ LCPUFA, eicosapentaenoic acid, docosahexaenoic acid, inflammation, anti inflammatory properties.
\end{abstract}

Este trabajo fue recibido el 17 de Mayo de 2011 y aceptado para ser publicado el 8 de Agosto de 2011.

\section{INTRODUCCIÓN}

Los ácidos grasos poliinsaturados de cadena larga (AGPICL) son componentes dietarios que participan en múltiples procesos fisiológicos, donde cumplen un rol estructural en los fosfolípidos de las membranas celulares y son sustratos para la síntesis de diversos mediadores fisiológicos. Dentro de los AGPICL encontramos dos grupos principales; los ácidos grasos omega-3 ( $\omega-3)$ y omega-6 ( $\omega-6)$, los cuales son ácidos grasos esenciales (AGE) para el ser humano debido a que carecemos de la maquinaria enzimática necesaria para biosintetizarlos (1). La nomenclatura “ $\omega$ ” considera como carbono principal al átomo de carbono del grupo metilo terminal del ácido graso (nomenclatura inversa a la IUPAC "International Union of Practical and Applied Chemistry") e identifica el primer doble enlace más cercano a este grupo químico. El primer exponente de los ácidos grasos omega-3 es el ácido $\alpha$-linolénico (C18:3) el cual vía desaturasas y elongasas se puede transformar en el ácido eicosapentaenoico (C20:5, EPA) y posteriormente en el ácido docosahexaenoico (C22:6, DHA). El primer exponente de los ácidos grasos $\omega-6$ es el ácido linoleico (C18:2) y uno de sus derivados más importantes es el ácido araquidónico $(\mathrm{C} 20: 4, \mathrm{AA})$ figura 
1 (2). Las fuentes alimentarias del ácido linoleico y del ácido $\alpha$-linolénico son los alimentos de origen vegetal, especialmente los aceites (soya, linaza, canola, entre otros.) y los frutos secos (almendra, nuez, maní, entre otros). La fuente nutricional de los AGPICL derivados de estos, son los alimentos de origen animal. El AA se encuentra en las carnes (vacuno, cordero y cerdo). El EPA y el DHA se encuentran tanto en animales como vegetales de origen marino, particularmente en pescados con un elevado contenido de grasa, como el atún, jurel, salmón, entre otros. (3). El AA, el EPA y el DHA son importantes componentes estructurales de los fosfolípidos de las membranas y son el sustrato para la formación de una serie de derivados lipídicos llamados eicosanoides (derivados de 20 átomos de carbono en el caso del AA y EPA) y docosanoides (derivados de 22 átomos de carbono, en el caso del DHA), los cuales ejercen importantes acciones en el metabolismo celular (4). El EPA y el DHA han sido el foco de interés de un gran número de investigaciones producto de sus bien caracterizados efectos antiinflamatorios y citoprotectores (5 - 8). En el presente artículo se analizan las múltiples aplicaciones clínicas y nutricionales y el potencial terapéutico de los AGPICL $\omega$-3 de origen marino (pescados grasos y/o microalgas) en diversas enfermedades. Además, se resumen los mecanismos por los cuales estos ácidos grasos ejercerían sus efectos benéficos en el organismo.

\section{AGPICL (1-3 E INFLAMACIÓN}

El AA al ser un componente de la estructura de las membranas celulares, es liberado desde los fosfolípidos por la activación de la enzima fosfolipasa $\mathrm{A}_{2}\left(\mathrm{FLA}_{2}\right)$ durante las primeras etapas de un proceso inflamatorio. Posteriormente, un grupo de enzimas conocidas como lipooxigenasas y ciclooxigenasas metabolizan al AA generando eicosanoides bioactivos, entre los que se encuentran las prostaglandinas, leucotrienos y tromboxanos (9), (figura 2). El AA tiene dos vías distintas de acción, la primera vía involucra a las ciclooxigenasas, las que convierten al AA en el tromboxano $\mathrm{A}_{2}\left(\mathrm{TXA}_{2}\right)$ y en varias prostaglandinas (10). Es importante destacar que existen dos isoenzimas distintas de la ciclooxigenasa: 1a ciclooxigenasa-1 (COX-1) y la ciclooxigenasa-2 (COX2). La COX-1 se expresa como componente constitutivo

\section{FIGURA 1}

Metabolización de ácidos grasos poliinsaturados $\omega$-6 y $\omega$-3. Vías de desaturación y elongación de los ácidos linoleico y $\alpha$-linólénico.

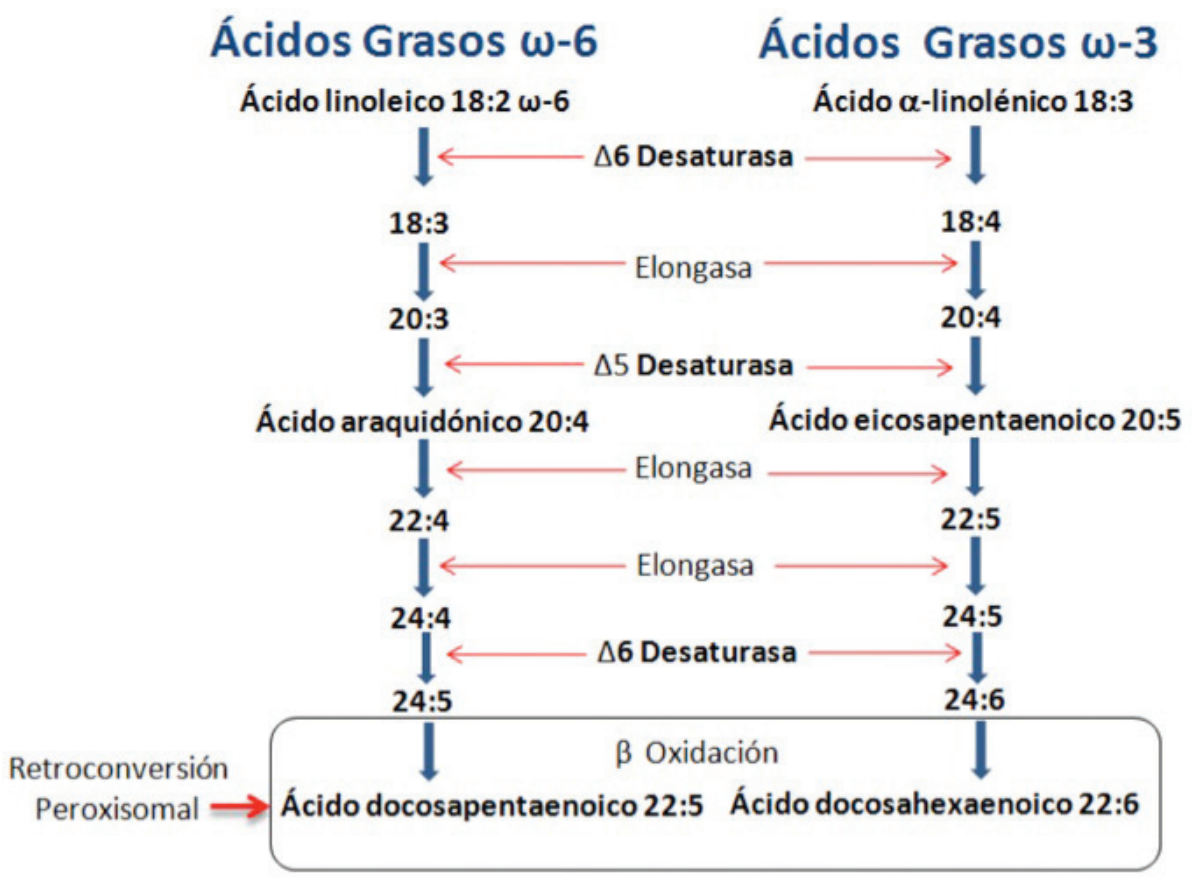


en la mayoría de las células; la COX-2 es inducible en diferentes tipos de células y su expresión se incrementa por diversos estímulos $(4,5)$. Entre las prostaglandinas derivadas del $\mathrm{AA}$, la prostaglandina $\mathrm{E}_{2}\left(\mathrm{PGE}_{2}\right)$, es un potente mediador de la inflamación, el dolor, la fiebre y del aumento de la permeabilidad vascular (11). La segunda vía del AA involucra la enzima 5-lipoxigenasa y la formación de distintos leucotrienos, entre los que destacan el leucotrieno $\mathrm{B}_{4}\left(\mathrm{LTB}_{4}\right)$, el leucotrieno $\mathrm{C}_{4} \mathrm{y}$ el leucotrieno $\mathrm{D}_{4}$, los cuales son potentes agentes proinflamatorios que aumentan la permeabilidad vascular, la actividad de las células inmunes, y estimulan la liberación de citoquinas inflamatorias (12).

En los humanos y en animales las dietas ricas en EPA y DHA aumentan la proporción de estos ácidos grasos en las membranas celulares, particularmente en los linfocitos lo cual, además de reducir el contenido de AA en las membranas de estas células por un efecto de competencia, disminuye la generación de los productos pro-inflamatorios derivados del AGPICL $\omega-6(13,14)$. El EPA también es un sustrato de la COX (1 y 2) y de la lipooxigenasa-5 cuando se ubica en la membrana plasmática, por lo cual compite con el AA en la generación de eicosaniodes, pero en el caso del EPA estos presentan propiedades antiinflamatorias. El EPA inhibe in vitro la transformación del AA por la COX en sus derivados eicosanoides $(14,15)$ con lo cual la suplementación dietaria con EPA puede reducir la formación de $\mathrm{PGE}_{2}, \mathrm{TXA}_{2} \mathrm{y}$
$\mathrm{LTB}_{4}$ y mantener los niveles de la prostaglandina $\mathrm{I}_{2}$ (una prostaciclina) la cual es un inhibidor de la agregación plaquetaria $(16,17)$. Si bien los productos del metabolismo del AA ( $\mathrm{PGE}_{2}, \mathrm{TXA}_{2}$ y $\left.\mathrm{LTB}_{4}\right)$ tienen propiedades pro-inflamatorias, los productos de la conversión del EPA ( TXA $_{3}$, prostaglandinas $\mathrm{I}_{3}$ y $\mathrm{E}_{3}$ y $\mathrm{LTB}_{5}$ ) son significativamente menos potentes en estimular la inflamación, la vasoconstricción y la agregación plaquetaria, e incluso pueden antagonizar los efectos típicamente pro-inflamatorios de los eicosanoides derivados del AA (18), (figura 3).

La suplementación dietaria con EPA y DHA también es capaz de reducir la producción de citoquinas pro-inflamatorias, tales como la interleuquina-1, la interleuquina-6, la interleuquina- 8 y el factor de necrosis tumoral- $\alpha$ (TNF- $\alpha$ ), que se liberan cuando los macrófagos y monocitos son activados (19). Si bien estas citoquinas son potentes activadores de la función inmune, el exceso de actividad de estas sustancias contribuye a la inflamación patológica, situación observada en la inflamación intestinal crónica (20), en la artritis reumatoidea (21), entre otras patologías inflamatorias. El TNF- $\alpha$ tiene un rol importante en el desarrollo de caquexia en pacientes con cáncer (22). En este sentido la suplementación dietaría con EPA y DHA puede reducir la producción de citoquinas inflamatorias y los efectos del TNF- $\alpha$ (23).

La producción de citoquinas inflamatorias está re-

\section{FIGURA 2}

Bioconversión y efectos metabólicos de eicosanoides derivados del ácido araquidónico (AA) y ácido eicosapentaenoico (EPA).

\section{Ácido Linoleico $\Longrightarrow$ Ácido Araquidónico Ácido Eicosapentaenoico $\rightleftharpoons$ Ácido a-linolénico}

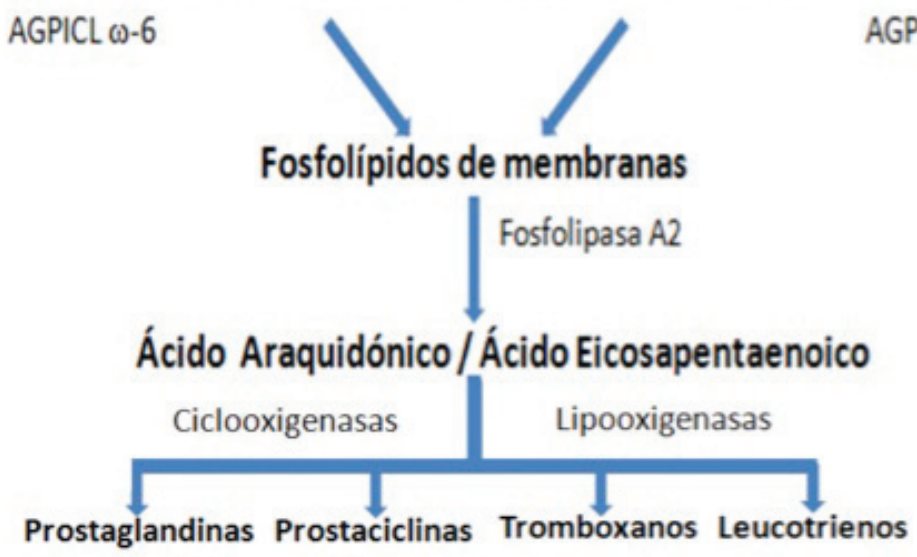


gulada por la disponibilidad de eicosanoides derivados del AA, lo cual puede ser modulado por la ingesta de AGPICL $\omega-3$, los que incluso actúan a nivel génico, ya que la expresión de los genes para citoquinas y moléculas de adhesión celular se reduce en respuesta a la exposición a AGPICL $\omega$-3 (24). Además, los AGPICL $\omega$-3 afectan directamente las vías de señalización intracelular asociadas con la activación de factores de transcripción, como el factor nuclear $x \mathrm{~B}(\mathrm{NF}-\mathrm{kB})$ y los factores de proliferación peroxisomal (PPARs) (25) que regulan la expresión de una serie de genes cuyos productos son pro-inflamatorios (26).

La inflamación es una característica clave en una serie de condiciones clínicas, como las enfermedades cardiovasculares, neurodegenerativas, cáncer, inflamación intestinal crónica, artritis reumatoidea, asma entre muchas otras patologías (15), por lo cual los AGPICL $\omega-3$ son candidatos terapéuticos ideales para la prevención y/o el tratamiento de patologías donde la inflamación juega un rol central.

\section{AGPICL $\omega$-3 Y ENFERMEDADES CARDIOVASCULARES}

Los primeros datos que evidenciaron los efectos cardioprotectores de los AGPICL $\omega$-3 surgieron a partir de los estudios realizados en los esquimales (inuits), quienes a pesar de tener una elevada ingesta de grasas (superior al 30\% de los requerimientos energéticos) presentaban una muy baja incidencia de enfermedades cardiovasculares, identificándose como la fuente dietaria de estas grasas los animales de origen marino (mamíferos y peces ricos en estos lípidos) (27). Estos resultados fueron confirmados en estudios realizados posteriormente en poblaciones con una alimentación similar, las cuales evidenciaron, además de una baja incidencia de enfermedades cardiovasculares, una menor manifestación de enfermedades inflamatorias (28). Dentro de las enfermedades cardiovasculares, la aterosclerosis es un proceso fisiopatológico de origen multifactorial de desarrollo a largo plazo. En este proceso destacan dos componentes principales; la dislipidemia (triglicéridos y colesterol elevados) y la inflamación. La reducción de los lípidos plasmáticos, especialmente los triglicéridos (TG) generada por el consumo de AGPICL $\omega-3$, es uno de los efectos con mayor evidencia tanto en humanos como en animales (29). Los aceites de pescado han demostrado que disminuyen el colesterol plasmático y los niveles de TG a través de la inhibición de la biosíntesis de lipoproteínas de muy baja densidad (VLDL) y de TG en el hígado, sin alterar la biosíntesis de lipoproteínas de alta densidad (HDL) (30). El efecto sobre los niveles plasmáticos de TG, HDL y LDL sería inverso cuando se ingieren aceites ricos en AGPICL $\omega-6$ (31), indicando que la relación ácidos grasos $\omega-3 / \omega-6$ dietaria operaría como un sensor hepático para la regulación del metabolismo lipídico. Los efectos benéficos de los AGPICL

\section{FIGURA 3}

Efectos metabólicos de los tromboxanos, prostaciclinas y leucotrienos derivados del ácido araquidónico (AA) y ácido eicosapentaenoico (EPA).

\begin{tabular}{|c|c|c|c|c|c|}
\hline \multicolumn{2}{|c|}{ PLAQUETAS } & \multicolumn{2}{|c|}{ CÉLULASENDOTELIALES } & \multicolumn{2}{|c|}{ LEUCOCITOS } \\
\hline$A A$ & EPA & $A A$ & EPA & $A A$ & EPA \\
\hline \multicolumn{2}{|c|}{ Via ciclooxigenasas } & \multicolumn{2}{|c|}{ Vía ciclooxigenasas } & \multicolumn{2}{|c|}{ Vía lipooxigenasas } \\
\hline $\begin{array}{l}\text { Tromboxano } \mathrm{A}_{2} \\
\text { Agregación } \\
\text { Plaquetaria } \\
\text { Vasocontrictor }\end{array}$ & $\begin{array}{l}\text { Tromboxano } \mathrm{A}_{3} \\
\text { Biológicamente } \\
\text { inactivo }\end{array}$ & $\begin{array}{l}\text { Postaciclina }{ }_{2} \\
\text { Vasodilatador } \\
\text { Antiagregación } \\
\text { Plaquetaria }\end{array}$ & $\begin{array}{l}\text { Prostaciclina I }_{3} \\
\text { Vasodilatador } \\
\text { Antiagregación } \\
\text { Plaquetaria }\end{array}$ & $\begin{array}{l}\text { Leucotrieno } \mathrm{B}_{4} \\
\text { Proinflamatorio } \\
\text { Quimiotáxico } \\
\text { Adhesión } \\
\text { celular }\end{array}$ & $\begin{array}{l}\text { Leucotrieno } \mathrm{B}_{5} \\
\text { Antiinflamatorio } \\
\text { No quimiotáxico } \\
\text { Inhibe adhesión }\end{array}$ \\
\hline
\end{tabular}


$\omega$-3 sobre las enfermedades cardiovasculares han sido abundantemente documentados en estudios realizados en humanos y animales $(17,32,33)$. Todos estos efectos se atribuyen al EPA y sus derivados metabólicos (17, 32), aunque actualmente se plantea que el DHA sería un agente cardioprotector más potente que el EPA (34).

La ingesta de aceite de pescado reduce la ocurrencia de lesiones ateroescleróticas, disminuye la frecuencia de paros cardíacos y reduce la mortalidad global en pacientes con riesgo de enfermedad cardiovascular (35). Además de mejorar el perfil lipídico, los AGPICL $\omega-3$ ejercerían leves disminuciones en la presión arterial (32). La reducción de los TG, el aumento del colesterol HDL, la reducción de la inflamación vascular y la disminución de la agregación plaquetaría, favorecerían dicha disminución en la presión arterial, aunque los mecanismos específicos de este efecto aún no están descritos completamente (36).

Los AGPICL $\omega-3$ también parecen ejercer una serie de efectos potencialmente beneficiosos sobre la musculatura vascular lisa, mediante la reducción de la pérdida de calcio intracelular y en la disminución de la proliferación de células musculares lisas (a través de la inhibición de factores de crecimiento) y el aumento de la producción de óxido nítrico (37). Además de sus efectos sobre la dislipidemia y la arteriosclerosis, los AGPICL $\omega-3$ podrían tener efectos antiarrítmicos (38).

La acción reguladora del trabajo cardíaco de estos ácidos grasos estaría relacionada con su capacidad para inhibir los canales de calcio tipo-L en las células cardíacas, lo que a su vez prolongaría el período refractario haciendo al miocardio menos susceptible a las arritmias potencialmente peligrosas (36 - 38). En un estudio realizado en miocitos cardíacos, se observó que el EPA puede tener efectos protectores contra la hipertrofia cardíaca mediante la inhibición de la endotelina-1 (hormona vascular que participa en el proceso de hipertrofia de los miocitos) (39). El EPA y el DHA se almacenan rápidamente en los fosfolípidos de la membrana, especialmente en las células cardíacas, lo cual sería de especial utilidad clínica en pacientes que han sufrido un infarto (34). La evidencia clínica y epidemiológica nos indica que personas que consumen pescado, al menos una vez por semana, tienen una menor tasa de enfermedad cardiovascular (40).

Los efectos beneficiosos en la salud cardiovascular atribuidos a los AGPICL $\omega-3$, serían el resultado de los siguientes mecanismos: (i) disminución de los niveles plasmáticos de TG y del colesterol LDL, (ii) aumento del colesterol HDL, (iii) disminución de la presión arterial, (iv) reducción de la agregación plaquetaria y (v) disminución de incidencia de arritmias. Un estudio que consideró pacientes de diferentes países pudo establecer con mayor detalle los efectos beneficiosos de los AGPICL $\omega-3$, concluyendo que pacientes que consumían pescado, o algún suplemento nutricional con AGPICL $\omega-3$, presentaban una reducción en la tasa de mortalidad total, de mortalidad por problemas cardíacos y una disminución de infartos al miocardio (41). Considerando que los AGPICL $\omega$-3 no son solo el EPA y DHA, es necesario citar que con la administración de ALA (precursor del EPA y DHA) no se logran los mismos efectos benéficos que con sus derivados de mayor tamaño de cadena e insaturación (42).

También es importante destacar que el tipo y forma de preparación del pescado determina los efectos cardioprotectores de los AGPICL $\omega-3$. El consumo de pescados ricos en AGPICL $\omega-3$ (atún, jurel, salmón, entre otros) produce una significativa disminución en el riesgo de presentar isquemia cardíaca en sujetos mayores de 65 años; este efecto se observa cuando el pescado es consumido asado o al horno, mientras que cuando se consume frito no se observa dicho efecto (43).

\section{AGPICL (0-3 Y NEUROPROTECCIÓN}

Desde hace ya varios años estudios en humanos (44), en animales (45) y en modelos celulares in vitro (46), han revelado un efecto neuroprotector de los AGPICL $\omega-3$, especialmente en lesiones inducidas por isquemia y por excitotoxicidad producida por neurotransmisores (glutamato, principalmente) (47). En animales con diabetes mellitus, la suplementación dietaria con AGPICL $\omega-3$ (específicamente DHA) permitió prevenir el deterioro anatomofuncional en las neuronas, cuadro característico de la neuropatía diabética (48), y también reducir el daño oxidativo y los problemas de aprendizaje (modelo en laberinto de Morris) en ratas con lesión cerebral traumática (49). A su vez, ratas alimentadas con una dieta pobre en DHA, presentan trastornos de aprendizaje y de la función cognitiva, efectos que se revierten al suplementar con DHA (50).

Otras investigaciones se han centrado en los efectos neuroprotectores de los AGPICL $\omega-3$ en la enfermedad de Alzheimer, debido a que los pacientes de esta enfermedad tienen bajos niveles de DHA plasmáticos y en sus membranas celulares (51). En un modelo animal (ratón con enfermedad Alzheimer) al administrar una dieta enriquecida con AGPICL $\omega-3$ (EPA + DHA) se observó una reducción en la acumulación del péptido $\beta$-amiloide (péptido con acciones neurotóxicas) en más de un $70 \%$ de los casos (52).

Los efectos neuroprotectores de los AGPICL $\omega-3$ se deben a múltiples factores y pueden estar relacionados con una serie de efectos moleculares a nivel neuronal, 
especialmente en el sistema nervioso central (SNC). Por ejemplo in vitro, los AGPICL $\omega-3$ han demostrado ser capaces de prevenir la acumulación neuronal de calcio, bloqueando una señal que puede desencadenar una cascada de eventos celulares que inducen lesión y apoptósis neuronal (53).

Si bien los AGPICL $\omega-3$, por su estructura química (con numerosos dobles enlaces), son más vulnerables al estrés oxidativo (desarrollo de oxidación), en las células en general y especialmente las neuronas, pueden reducir el daño ocasionado por el estrés oxidativo a través de las neuroprotectinas (docosanoides derivados del DHA) (54). Además, los AGPICL $\omega$-3 pueden regular la expresión de genes neuroprotectores, como es el caso de la expresión del gen antiapoptotico Bcl2 (55). Los efectos neuroprotectores de los AGPICL $\omega-3$ en pacientes con neuropatía diabética pueden ser atribuibles a varias causas, incluido; (i) el mantenimiento del flujo sanguíneo al tejido nervioso, (ii) la preservación de la actividad de la bomba sodio-potasio ATPasa, (iii) cambios en la composición lipídica de la membrana neuronal y (iv) modificación del metabolismo de los lípidos en las neuronas (56). Actualmente se postula que los AGPICL $\omega-3$, especialmente el DHA, pueden ser utilizados como parte del tratamiento de múltiples neuropatologías, además de la neuropatía diabética y la enfermedad de Alzheimer, entre las que destacan la enfermedad de Parkinson (57), la esclerosis múltiple (58), la depresión (59) y la esquizofrenia (60).

\section{AGPICL (0-3 Y CÁNCER}

Un área de gran interés para el potencial uso clínico de los AGPICL $\omega$-3 es el cáncer y la caquexia relacionada con el cáncer. Estudios en ratones y en cultivos de células han demostrado que las dietas que contienen EPA y DHA retrasan tanto el crecimiento, la metástasis de los tumores primarios y los implantes de carcinoma humano en células mamarias (61-63). En la misma dirección, el uso de aceite de pescado como suplemento nutricional también aumenta la eficacia de los agentes quimioterapéuticos, como es el caso de la doxorrubicina en mujeres con cáncer mamario (64).

La suplementación con AGPICL $\omega-3$ induce la apoptosis y la diferenciación celular, así como la reducción de la proliferación celular en cultivos de células neoplásicas (65). En un estudio en ratas con cáncer de colon, se demostró que los AGPICL $\omega$-3 bloquean la formación de tumores inducida por fármacos, mediante el aumento de la diferenciación celular y la apoptosis en las primeras etapas de formación del tumor (66).

Otro estudio en ratas con cáncer de colon, demostró que los AGPICL $\omega-3$ afectan directamente la expresión de genes relacionados con la tumorogénesis y la apoptosis, reduciendo el daño celular y al DNA (67). Los AGPICL $\omega-3$ modulan también las principales moléculas de señalización intracelular y receptores nucleares, tales como PPAR $\alpha$ y LXRs, moléculas que al parecer desempeñan un papel en la regulación del crecimiento y la diferenciación de las células cancerígenas (67-69). La inhibición de la producción de prostaglandinas por los AGPICL $\omega$-3 también puede ayudar a modular el crecimiento tumoral y la apoptosis celular (70).

La caquexia, es un síndrome complejo de anorexia, pérdida de peso y pérdida de tejido (especialmente muscular), que se observa en muchos pacientes con cáncer. La presencia de caquexia es un indicador de mal pronóstico que complica el tratamiento farmacológico del cáncer y aumenta la toxicidad de los medicamentos. En este sentido se ha propuesto que el uso de suplementos nutricionales ricos en AGPICL $\omega-3$ permitiría aumentar el apetito, disminuir la pérdida de peso, aumentando la masa magra, proporcionando así una mejor calidad de vida al paciente $(71,72)$. La reducción plasmática de las citoquinas proinflamatorias, producida por el consumo de AGPICL $\omega$-3, beneficiaría claramente la sintomatología de los pacientes con caquexia relacionada a un cáncer, dado que un aumento en los niveles plasmáticos de citoquinas proinflamatorias se ha asociado con anorexia, pérdida de peso y con el hipermetabolismo asociado al cáncer (73-75). Sin embargo, el uso de AGPICL $\omega$-3 para tratar la caquexia en pacientes con cáncer aún esta en discusión.

\section{AGPICL (0-3 Y ENFERMEDAD INTESTINAL INFLAMATORIA}

La etiología de las enfermedades intestinales inflamatorias, como la enfermedad de Crohn y la colitis ulcerosa, es compleja y aún no se conocen los mecanismos específicos involucrados en su desarrollo. En este sentido; estudios epidemiológicos han sugerido que dietas ricas AGPICL $\omega-6$ pueden contribuir al desarrollo de estas patologías (76) considerando, además, que en los pacientes con patología intestinal inflamatoria se ha observado que presentan bajos niveles de AGPICL $\omega-3$, lo que podría contribuir a una mayor progresión de estas patologías (77). En el desarrollo de la patología intestinal inflamatoria juegan un rol central mediadores de la respuesta inflamatoria, tales como las interleuquinas (IL-1 y IL-6) y el factor de necrosis tumoral alfa (TNF- $\alpha$ ), siendo estos mediadores uno de los objetivos de la terapia farmacológica para estas patologías (76, 77). Si bien las actuales terapias farmacológicas han demostrado ser eficientes en reducir las recaídas en pacientes con patología intestinal inflamatoria, podrían 
generar diversos efectos secundarios a largo plazo, especialmente una disminución en la respuesta inmune (78). Los efectos de los AGPICL $\omega-3$ en la reducción de los niveles de citoquinas proinflamatorias demuestran que estos ácidos grasos son importantes agentes a considerar en el tratamiento de la patología inflamatoria intestinal (79). En estudios en animales con patología inflamatoria intestinal se ha demostrado que los AGPICL $\omega-3$ pueden reducir sustancialmente la producción de PGE, TNF- $\alpha$, $\mathrm{LTB}_{4}$ y $\mathrm{TXA}_{2}(80,81)$. En ratas con colitis ulcerosa, alimentadas con aceite de pescado, se observó una reducción en los niveles de mediadores inflamatorios y una mejoría en varios marcadores de daño celular (82). Si bien los resultados de algunos estudios sobre el uso de AGPICL $\omega-3$ en pacientes con patologías inflamatorias intestinales han permitido reducir las tasas de recaídas, una mejoría en los síntomas y una reducción del estrés oxidativo en el intestino (83), aún se requiere realizar mayores investigaciones con el propósito de obtener mayor certeza experimental y clínica sobre estos efectos benéficos.

\section{ARTRITIS REUMATOIDEA}

La artritis reumatoidea (AR) corresponde a un complejo desorden autoinmune que se caracteriza por una marcada inflamación y destrucción progresiva de los tejidos que forman las articulaciones, donde las citoquinas liberadas por linfocitos $\mathrm{T}$ activos tienen un un papel importante en los procesos inflamatorios asociados a esta enfermedad $(84,85)$. El tratamiento de la AR actualmente se centra el uso de fármacos antiinflamatorios e inmunosupresores, los cuales tienen efectos secundarios adversos a largo plazo (86). En este sentido, los estudios sobre el potencial efecto antiinflamatorio de los AGPICL $\omega-3$ han sido permanentes desde la década de 1980, cuando surgieron las primeras evidencias sobre el potencial antiinflamatorio de estos ácidos grasos. Estudios realizados tanto en humanos como animales han demostrado que la suplementación con AGPICL $\omega$-3 mediante aceites de pescado, reduce significativamente (hasta un $42 \%$ ) los niveles séricos de las IL-1, 2, 6 y 8, así como del TNF- $\alpha$ y de los LTB (87 - 90). La reducción de estos mediadores de la inflamación se asocia a una mejoría en los síntomas de la artritis reumatoidea, destacando una reducción de la tensión y la rigidez articular $(91,92)$. A su vez, dos estudios realizados en pacientes con AR que recibieron suplementos nutricionales de AGPICL $\omega-3$ (aceite de pescado), permitieron una reducción significativa del uso de antiinflamatorios no esteroidales (AINES) y de otros medicamentos antiinflamatorios $(93,94)$. Estos antecedentes permiten establecer un potencial uso de los AGPICL $\omega$-3 como parte del tratamiento de la AR, especialmente en las etapas tempranas de la enfermedad.

\section{DAÑO INDUCIDO POR ISQUEMIA - REPERFUSIÓN}

El daño por isquemia-reperfusión (IR) es una situación clínica que se presenta en diferentes órganos y tejidos, observándose lesiones por IR en infarto al miocardio, lesión renal aguda, apoplejías, e isquemia por shock sistémico (95). La administración de AGPICL $\omega-3$ como el EPA y el DHA, ha sido descrita como una estrategia preacondicionante frente al daño por IR cardíaca (96) y cerebral (97). Recientemente, nuestro grupo demostró que en un protocolo quirúrgico experimental de IR hepática en ratas, la administración de EPA y DHA permite disminuir significativamente el daño inducido por la IR hepática, donde es posible observar una buena preservación anátomo - funcional de los tejidos (26). Frente al daño inducido por la IR, los efectos citoprotectores de los AGPICL $\omega-3$ se asociarían a la capacidad de estos ácidos grasos para disminuir la respuesta inflamatoria e incrementar la actividad antioxidante, donde los AGPICL $\omega$-3 inhibirían la translocación al núcleo del NF-kB y su unión al DNA, produciendo así una reducción en la expresión de citoquinas pro-inflamatorias (25). Por otro lado, se ha descrito que la activación ejercida por los AGPICL $\omega$-3 sobre el factor de transcripción PPAR- $\alpha$, generaría complejos inactivos PPAR- $\alpha$ /NF-kBp65, disminuyendo la actividad de unión a DNA del NF-kBp50p65 (dimero funcional) (98), siendo el posible y más claro mecanismo mediante el cual los AGPICL $\omega-3$ ejercerían un efecto antiinflmatorio y citoprotector frente a la injuria generada por la IR $(25,98)$.

\section{CONCLUSIONES}

Los AGPICL $\omega$-3 de origen marino, como el EPA y el DHA, han demostrado ser eficaces en el tratamiento y prevención de variadas enfermedades, tales como cardiovasculares, neurodegenerativas, cáncer, enfermedad inflamatoria intestinal, artritis reumatoidea e injuria por isquemia/reperfusión. Estos ácidos grasos participarían directamente en la modulación de la respuesta inmune, disminuyendo la inflamación y el daño anatomo - funcional generado por esta, demostrándose el efecto antiinflamatorio y citoprotector de los AGPICL $\omega$-3. En este sentido las investigaciones futuras deberán centrar sus esfuerzos en establecer las dosis necesarias de estos ácidos grasos para lograr los efectos saludables descritos en esta revisión.

A nivel alimentario - nutricional las estrategias 
deberán orientarse a aumentar el consumo de los AGPICL $\omega-3$ en la población, especialmente si se considera que la dieta occidental es pobre en ellos, para lo cual se deberá fomentar el consumo de alimentos ricos EPA y DHA, principalmente pescados grasos, o desarrollar alimentos funcionales que los contengan en concentraciones terapéuticamente útiles, además de considerar el consumo complementario de suplementos nutricionales (nutracéuticos) con AGPICL $\omega-3$.

\section{RESUMEN}

Los ácidos grasos poliinsaturados de cadena larga omega-3 (AGPICL $\omega$-3) como el ácido eicosapentaenoico (EPA) y docosahexaenoico (DHA) se encuentran en cantidades importantes en los pescados grasos (atún, jurel y salmón) y especialmente en el aceite obtenido de estas especies, el cual actualmente se utiliza como suplemento nutricional (nutracéutico). Tanto el EPA como el DHA, luego de ser ingeridos, se incorporan rápidamente a los fosfolípidos de las membranas celulares donde pueden ser liberados por enzimas lipooxigenasas y ciclooxigenasas, originando productos con potentes propiedades citoprotectoras y especialmente antiinflamatorias. La evidencia clínica y epidemiológica de múltiples estudios permite establecer que el consumo de EPA y DHA puede contribuir a la prevención y/o tratamiento de una serie de patologías, especialmente aquellas donde la inflamación juega un papel preponderante en su desarrollo. El EPA y el DHA presentan propiedades antiinflamatorias, vía la generación ya sea de agentes anti-inflamatorios, como las resolvinas, o a través del bloqueo de agentes pro-inflamatorios. En el presente artículo se presentan evidencias sobre las posibles aplicaciones clínicas de los AGPICL $\omega-3$ en patologías tales como las enfermedades cardiovasculares, neurodegenerativas, cáncer, enfermedad inflamatoria intestinal, artritis reumatoidea e injuria por isquemia - reperfusión. La evidencia sugiere que los AGPICL $\omega-3$ pueden tener promisorias aplicaciones en el tratamiento y/o la prevención de diferentes patologías clínicas o nutricionales.

Palabras clave: AGPICL $\omega-3$, ácido eicosapentaenoico, ácido docosahexanoico, inflamación, propiedades antiinflamatorias.

Dirigir la correspondencia a:

Profesor

Rodrigo Valenzuela B.

Escuela de Nutrición y Dietética

Facultad de Medicina, Universidad de Chile

Santiago, Chile.

Casilla 1227. Independencia,

Santiago, Chile.
Teléfono: 56-2-9786014

Fax: 56-2-9786182

E-mail:tvalenzuelab@med.uchile.cl

\section{BIBLIOGRAFÍA}

1. Burr GO, Burr MM. On the nature and role of fatty acids essential in nutrition. J Biol Chem 1930;86:587-621.

2. Cunnane SC. Problems with essential fatty acids: time for a new paradigm? Prog Lipid Res 2003;42:544-68.

3. Simopoulos AP. Genetic variants in the metabolism of omega- 6 and omega- 3 fatty acids: their role in the determination of nutritional requirements and chronic disease risk. Exp Biol Med (Maywood) 2010;235:785-95.

4. Serhan $\mathrm{CN}$ and Chiang N. Endogenous pro-resolving and anti-inflammatory lipid mediators: a new pharmacologic genus. Br J Pharmacol 2008;153 Suppl 1:S200-15.

5. Calder PC. n-3 Polyunsaturated fatty acid, inflammation, and inflammatory disease. Am J Clin Nutr 2006; 83(suppl 6):1505S-19S.

6. Trebble TM, Wooten SA, Miles EA, Mullee M, Arden NK, Ballinger AB, et al. Prostaglandin E2 production and $\mathrm{T}$ cell function after fish-oil supplementation: response to antioxidant co-supplementation. Am J Clin Nutr 2003;78:376-82.

7. Simopoulos AP. Omega-3 fatty acids in inflammation and autoimmune disease. J Am Coll Nutr 2002; 21:495-505.

8. Wanten GJ, Calder PC. Immune modulation by parenteral lipid emulsions. Am J Clin Nutr 2007; 85:1171-84.

9. Calder PC, Yaqoob P, Thies F, Wallace FA, Miles EA. et al. Fatty acids and lymphocyte functions. $\mathrm{Br}$ J Nutr 2002;87:S31-48.

10. Holinstat M, Boutaud O, Apopa PL, Vesci J, Bala M, Oates JA, Hamm HE. Protease-activated receptor signaling in platelets activates cytosolic phospholipase A2 $\alpha$ differently for cyclooxygenase- 1 and 12-lipoxygenase catalysis. Arterioscler Thromb Vasc Biol 2011:31·435-42.

11. Sampath H, Ntambi JM. Polyunsaturated fatty acid regulation of genes of lipid metabolism. Annu Rev Nutr 2005;25:317-40.

12. Xi S, Cohen D, Barve S, Chen LH. Fish oil suppressed cytokines and nuclear factor kappa B induced by murine AIDS virus infection. Nutr Res 2001; 21:865-78.

13. Lapillone A, Clark SD, Heird WC. Polyunsaturated fatty acids and gene expression. Lipid metabolism 
and therapy. Curr Opin Clin Nutr Metab Care 2004; 7:151-6.

14. Sampath H, Ntambi JMPolyunsaturated fatty acid regulation of gene expression. Nutr Rev 2004; 62:333-9.

15. Wall R, Ross RP, Fitzgerald GF, Stanton C. Fatty acids from fish: the anti-inflammatory potential of long-chain omega-3 fatty acids. Nutr Rev 2010; 68:280-9.

16. Calder PC, Albers R, Antoine JM, Blum S, BourdetSicard R, Ferns GA, et al. Inflammatory disease processes and interactions with nutrition. Br J Nutr 2009;101 Suppl 1:S1-45.

17. Cottin SC, Sanders TA, Hall WL. The differential effects of EPA and DHA on cardiovascular risk factors. Proc Nutr Soc 2011; 24:1-17.

18. Layé $S$. Polyunsaturated fatty acids, neuroinflammation and well being. Prostaglandins Leukot Essent Fatty Acids 2010;82:295-303.

19. Camuesco D, Comalada M, Concha A, Nieto A, Sierra S, Xaus J, et al. Intestinal anti-inflammatory activity of combined quercitrin and dietary olive oil supplemented with fish oil, rich in EPA and DHA (n-3) polyunsaturated fatty acids, in rats with DSSinduced colitis. Clin Nutr 2006; 25:466-6.

20. Nieto N, Torres MI, Ríos A, Gil A. Dietary polyunsaturated fatty acids improve his-tological and biochemical alterations in rats with experimental ulcerative colitis. J Nutr 2002;132:11-9.

21. Hurst S, Zainal Z, Caterson B, Hughes CE, Harwood JL. Dietary fatty acids and arthritis. Prostaglandins Leukot Essent Fatty Acids 2010;82:315-8.

22. Szymanski KM, Wheeler DC, Mucci LA. Fish consumption and prostate cancer risk: a review and meta-analysis. Am J Clin Nutr 2010;92:1223-33.

23. Lima-Salgado M, Sampaio C, Cury-Boaventura F, Curi R. Modulatory effect of fatty acids on fungicidal activity, respiratory burst and TNF- $\alpha$ and IL- 6 production in J774 murine macrophages. Br J Nutr 2011;105:1173-9.

24. Mickleborough TD, Tecklenburg SL, Montgomery GS, Lindley MR. Eicosapentaenoic acid is more effective than docosahexaenoic acid in inhibiting proinflammatory mediator production and transcription from LPS-induced human asthmatic alveolar macrophage cells. Clin Nutr 2009; 28:71-7.

25. Huang F, Wei H, Luo H, Jiang S, Peng J. EPA inhibits the inhibitor of $\chi \mathrm{B} \alpha(\mathrm{I} \varkappa \mathrm{B} \alpha) / \mathrm{NF}-\varkappa \mathrm{B} /$ muscle RING finger 1 pathway in $\mathrm{C} 2 \mathrm{C} 12$ myotubes in a PPAR $\gamma$-dependent manner. Br J Nutr 2011; 105:348-356.

26. Zúñiga J, Venegas F, Villarreal M, Núñez D,
Chandía M, Valenzuela R, et al. Protection against in vivo liver ischemia-reperfusion injury by $n-3$ long-chain polyunsaturated fatty acids in the rat. Free Radic Res 2010;44:854-63.

27. Petrova S, Dimitrov P, Willett WC, Campos H. The global availability of n-3 fatty acids. Public Health Nutr 2011; 31:1-8.

28. Egert $\mathrm{S}$, Stehle P. Impact of n-3 fatty acids on endothelial function: results from human interventions studies. Curr Opin Clin Nutr Metab Care 2011; 14:121-31.

29. Nodari S, Triggiani M, Campia U, Manerba A, Milesi G, Cesana BM, et al. Effects of n-3 polyunsaturated fatty acids on left ventricular function and functional capacity in patients with dilated cardiomyopathy. J Am Coll Cardiol 2011;57:870-9.

30. Manerba A, Vizzardi E, Metra M, Dei Cas L. n-3 PUFAs and cardiovascular disease prevention. Future Cardiol 2010; 6:343-50.

31. Roth EM, Harris WS. Fish oil for primary and secondary prevention of coronary heart disease. Curr Atheroscler Rep 2010;12:66-72.

32. Wang C, Harris WS, Chung M, Lichtenstein AH, Balk EM, Kupelnick B et al. n-3 fatty acids from fish or fish oil supple-ments, but not alpha-linolenic acid, benefit cardiovascular disease outcomes in primary and secondary prevention studies: a systematic review. Am J Clin Nutr 2006; 84:5-17.

33. Slee EL, McLennan PL, Owen AJ, Theiss ML. Low dietary fish-oil threshold for myocardial membrane n-3 PUFA enrichment independent of n-6 PUFA intake in rats. J Lipid Res 2010; 51:1841-8.

34. Borghi C, Cicero AF. Recent evidence on the role of omega-3 polyunsaturated fatty acids on blood pressure control and hypertension-related complications. Future Lipidology 2006; 1:569-77.

35. Rodrigo R, Cereceda M, Castillo R, Asenjo R, Zamorano J, Araya J, et al. Prevention of atrial fibrillation following cardiac surgery: basis for a novel therapeutic strategy based on non-hypoxic myocardial preconditioning. Pharmacol Ther 2008;118:104-27.

36. Leaf A, Albert CM, Josephson M, David Steinhaus, Kluger J, Kang J, et al. Prevention of fatal arrhythmias in high-risk subjects by fish oil n-3 fatty acid intake. Circulation 2005; 112:2762-8.

37. Adkins Y, Kelley DS. Mechanisms underlying the cardioprotective effects of omega-3 polyunsaturated fatty acids. J Nutr Biochem 2010; 21:781-92.

38. Metcalf RG, James MJ, Gibson RA, Edwards J, Stubberfield J, Stuklis R, et al. Effects of fish oil supplementation on myocardial fatty acid in hu- 
mans. Am J Clin Nutr 2007; 85:1222-28.

39. Brenna JT. Efficiency of conversion of alphalinolenic acid to long chain n-3 fatty acids in man. Curr Opin Clin Nutr Metab Care 2002; 5:127-32.

40. Shimojo N, Jesmin S, Zaedi S, Maeda S, Soma M, Aonuma K, Eicosapentaenoic acid prevents endothelin-1-induced cardiomyocyte hypertrophy in vitro through the suppression of TGF-beta 1 and phosphorylated JNK. Am J Physiol Heart Circ Physiol 2006; 291:H835-45.

41. Manerba A, Vizzardi E, Metra M, Dei Cas L. n-3 PUFAs and cardiovascular disease prevention. Future Cardiol 2010;6:343-50.

42. Mozaffarian D.Fish and n-3 fatty acids for the prevention of fatal coronary heart disease and sudden cardiac death. Am J Clin Nutr 2008;87:1991S1996S.

43. Geleijnse JM, de Goede J, Brouwer IA. Alpha-linolenic acid: is it essential to cardiovascular health? Curr Atheroscler Rep 2010; 12:359-67.

44. Mozaffarian D, Lemaitre RN, Kuller LH, Burke GL, Tracy RP, Siscovick DS. Cardiac benefits of fish consumption may depend on the type of fish meal consumed: the Cardiovascular Health Study. Circulation 2003;107:1372-7.

45. Lauritzen I, Blondeau N, Heurteaux C, Widmann C, Romey G, Lazdunski M. olyunsaturated fatty acids are potent neuroprotectors. EMBO J 2000; 19:1784-93.

46. Högyes E, Nyakas C, Kiliaan A, Farkas T, Penke B, Luiten PG. Neuroprotective effect of developmental docosahexaenoic acid supplement against excitotoxic brain damage in infant rats. Neuroscience 2003; 119:999-1012.

47. Ménard C, Patenaude C, Gagné AM, Massicotte G. AMPA receptor mediated cell death is reduced by docosahexaenoic acid but not by eicosapentaenoic acid in area CA1 of hippocampal slice cultures. J Neurosci Res 2009; 87:876-86.

48. Mayurasakorn K, Williams JJ, Ten VS, Deckelbaum RJ. Docosahexaenoic acid: brain accretion and roles in neuroprotection after brain hypoxia and ischemia. Curr Opin Clin Nutr Metab Care 2011;14:158-67.

49. Gerbi A, Maixent JM, Ansaldi JL et al. Fish oil supplementation prevents diabetes-induced nerve conduction velocity and neuroanatomical changes in rats. J Nutr 1999; 129:207-13.

50. Wu A, Ying Z, Gomez-Pinilla F. Dietary omega-3 fatty acids normalize BDNF levels, reduce oxidative damage, and counteract learning disability after traumatic brain injury in rats. J Neurotrauma 2004; 21:1457-67.
51. Catalan J, Moriguchi T, Slotnick B et al. Cognitive defects in docosahexaenoic acid-deficient rats. Behav Neurosci 2002; 116:1022-31.

52. Soderberg M, Edlund C, Kristensson K, Dallner G. Fatty acid composition of brain phospholipids in aging and in Alzheimer's disease. Lipids 1991; 26: 421-5.

53. Lim GP, Calon F, Morihara T, Yang F, Teter B, Ubeda O, Salem N Jr. A diet enriched with the omega-3 fatty acid do-cosahexaenoic acid reduces amyloid burden in an aged Alzheimer mouse model. J Neurosci 2005; 25:3032-40.

54. Zhang C, Bazan NG. Lipid mediated cell signaling protects against injury and neurodegeneration. $\mathrm{J}$ Nutr 2010; 140:858-63.

55. Bazan NG. Neuroprotectin D1-mediated anti-inflammatory and survival signaling in stroke, retinal degenerations, and Alzheimer's disease. J Lipid Res 2009;50 Suppl:S400-5.

56. Lukiw WJ, Cui JG, Marcheselli VL, Bodker M, Botkjaer A, Gotlinger K, et al. A role for docosahexaenoic acid-derived neuroprotectin D1 in neural cell survival and Alzheimer disease. J Clin Invest 2005;115:2774-83.

57. Bazan NG. Cell survival matters: docosahexaenoic acid signaling, neuroprotection and photoreceptors. Trends Neurosci 2006; 29:263-71.

58. Calon F, Cole G.Neuroprotective action of omega-3 polyunsaturated fatty acids against neurodegenerative diseases: evidence from animal studies. Prostaglandins Leukot Essent Fatty Acids 2007;77:287-93.

59. Mehta LR, Dworkin RH, Schwid SR. Polyunsaturated fatty acids and their potential therapeutic role in multiple sclerosis. Nat Clin Pract Neurol 2009;5:82-92.

60. Rondanelli M, Giacosa A, Opizzi A, Pelucchi C, La Vecchia C, Montorfano G, et al. Long chain omega 3 polyunsaturated fatty acids supplementation in the treatment of elderly depression: effects on depressive symptoms, on phospholipids fatty acids profile and on health related quality of life. J Nutr Health Aging 2011;15:37-44.

61. Peet M. Nutrition and schizophrenia: Beyond omega-3 fatty acids. Prostag Leukot Essent Fatty Acids 2004;70:417-22.

62. Rose DP, Connolly JM, Rayburn J, Rayburn J, Coleman M. Influence of diets containing eicosapentaenoic acid on growth and metastasis of breast cancer cells in nude mice. J Nat Cancer Inst 1995; 87:587-92.

63. Cavazos DA, Price RS, Apte SS, Degraffenried LA. Docosahexaenoic acid selectively induces human 
prostate cancer cell sensitivity to oxidative stress through modulation of NF- $x$ B. Prostate. 2011. doi: 10.1002/pros.21359.

64. Mandal CC, Ghosh-Choudhury T, Yoneda T, Choudhury GG, Ghosh-Choudhury N. Fish oil prevents breast cancer cell metastasis to bone. Biochem Biophys Res Commun 2010;402:602-7.

65. Hardman WE, Avula CP, Fernandes G et al. Three percent dietary fish oil concen-trate increased efficacy of doxorubicin against MDA-MB 231 breast cancer xenographs. Clin Cancer Res 2001; 7: 2041-9.

66. Yam D, Peled A, Schinitzky M. Suppression of tumor growth and metastasis by dietary fish oil combined with vitamins $\mathrm{E}$ and $\mathrm{C}$ and cisplatin. Cancer Chemother Pharmacol 2001; 47:34-40.

67. Vanamala J, Glagolenko A, Yang P, Carroll RJ, Murphy ME, Newman RA, et al. Dietary fish oil and pectin enhance colonocyte apoptosis in part through suppression of PPARdelta/PGE2 and elevation of PGE3. Carcinogenesis 2008; 29:790-6.

68. Fan YY, Spencer TE, Wang N et al. Chemopreventive n-3 fatty acids activate RXRalpha in colonocytes. Carcinogenesis 2003; 24:1541-8.

69. Gupta RA, Sarraf P, Brockman JA et al. Peroxisome proliferator-activated recep-tor gamma and transforming growth factor beta pathways inhibit intestinal epithelial cell growth by regulating levels of TSC-22. J Biol Chem 2003; 278:7431-8.

70. Wu M, Harvey KA, Ruzmetov N et al. Omega-3 polyunsaturated fatty acids attenuate breast cancer growth through activation of a neutral sphingomyelinase-mediated pathway. Int J Cancer 2005; 117:340-8.

71. Tsujii M, DuBois RN. Alterations in cel-lular adhesion and apoptosis in epithe-lial cells overexpressing prostaglandin endoperoxide synthase 2. Cell 1995; 83:493-501.

72. Barber MD, Ross JA, Voss AC, Tisdale MJ, Fearon KC. The effect of an oral nutritional supplement enriched with fish oil on weight loss in patients with pancreatic cancer. Br J Cancer 1999; 81:80-6.

73. Fearon KC, Von Meyenfeldt M, Moses AG et al. An energy and protein dense, high n-3 fatty acid oral supplement promotes weight gain in cancer cachexia. Eur J Cancer 2001; 37(suppl 6):S27-S28.

74. Fearon KC, Von Meyenfeldt MF, Moses AG et al. Effect of a protein and energy dense n-3 fatty acid enriched oral supple-ment on loss of weight and lean tissue in cancer cachexia: a randomised double blind trial. Gut 2003; 52:1479-86.

75. Deans C, Wigmore SJ. Systemic inflammation, ca- chexia and prognosis in patients with cancer. Curr Opin Clin Nutr Metabol Care 2005; 8:265-9.

76. Bruera E, Strasser F, Palmer JL Willey J, Calder K, Amyotte G,. Effect of fish oil on appetite and other symptoms in patients with advanced cancer and anorexia/cachexia: a double-blind placebo-controlled study. J Clin Oncol 2003; 21:129-34.

77. Calder PC. Polyunsaturated fatty acids, inflammatory processes and inflammatory bowel diseases. Mol Nutr Food Res 2008; 52(8):885-97.

78. Simopoulos AP. Omega-3 fatty acids in inflammation and autoimmune diseases. J Am Coll Nutr 2002;21(6):495-505.

79. Tamboli CP. Current medical therapy for chronic inflammatory bowel diseases. Surg Clin North Am 2007; 87(3):697-725.

80. Tsekos E, Reuter C, Stehle P and Boeden G. Perioperative administration of parenteral fish oil supplements in a routine clinical setting improves patient outcome after major abdominal surgery. Clin Nutr 2004; 23(3):325-30.

81. Kono H, Fujii H, Ogiku M, Tsuchiya M, Ishii K, and Hara M. Enteral diets enriched with medium-chain triglycerides and N-3 fatty acids prevent chemically induced experimental colitis in rats. Transl Res 2010;156:282-91.

82. Camuesco D, Gálvez J, Nieto A, Comalada M, Rodríguez-Cabezas ME, Concha A, et al. Dietary olive oil supplemented with fish oil, rich in EPA and DHA (n-3) polyunsaturated fatty acids, attenuates colonic inflammation in rats with DSS-induced colitis. J Nutr 2005;135:687-94.

83. Hassan A, Ibrahim A, Mbodji K, Coëffier M, Ziegler F, Bounoure F, Chardigny JM, Skiba M, Savoye G, Déchelotte P, Marion-Letellier R. An $\alpha$-linolenic acid-rich formula reduces oxidative stress and inflammation by regulating $\mathrm{NF}-x \mathrm{~B}$ in rats with TNBS-induced colitis. J Nutr 2010;140:1714-21.

84. Uchiyama K, Nakamura M, Odahara S, Koido S, Katahira K, Shiraishi H, et al. N-3 polyunsaturated fatty acid diet therapy for patients with inflammatory bowel disease. Inflamm Bowel Dis 2010; 16:1696-707.

85. Parameswaran N, and Patial S. Tumor necrosis factor- $\alpha$ signaling in macrophages. Crit Rev Eukaryot Gene Expr 2010;20:87-103.

86. McInnes IB, O'Dell JR. and State of the art: rheumatoid arthritis. Ann Rheum Dis 2010;69:1898-906.

87. Scott DL, Wolfe F, Huizinga TW. Rheumatoid arthritis. Lancet 2010;25: 1094-108.

88. Caughey GE, James MJ, Proudman SM, Cleland LG. Fish oil supplementation increases the cyclo- 
oxygenase inhibitory activity of paracetamol in rheumatoid arthritis patients. Complement Ther Med 2010;18:171-4.

89. Lerna M, Kerr A, Scales H, Berge K, Griinari M Supplementation of diet with krill oil protects against experimental rheumatoid arthritis. BMC Musculoskelet Disord 2010;29;(11):136.

90. Bahadori B, Uitz E, Thonhofer R, Trummer M Pestemer-Lach_L, McCarty M, Krejs GI bmega-3 Eatty acids infusjons as adjuvant therapy in rheumatoid arthritis. JPEN J Parenter Enteral Nutr 2010;34:151-5.

91. Hurst S, ZainalZ, Caterson B, Hughes CE, Harwood JL. Dietary fatty acids and arthritis. Prostaglandins Leukot Essent Fatty Acids 2010;82:315-8.

92. Cleland LG, French J, Betts HW., Murphy GA, Elliott MJ, Clinical and biochemical effects of dietary fish oil supplements in rheumatoid arthritis, J Rheumatol 1988;15:1471-5.

93. Dawczynski C, Schubert R, Hein G, Müller A, Eidner T, Vogelsang H, Basu S, Jahreis G. Longterm moderate intervention with n-3 long-chain PUFA-supplemented dairy products: effects on pathophysiological biomarkers in patients with rheumatoid arthritis. Br J Nutr 2009;101:1517-26.
94. Galarraga B, Ho M, Youssef HM, Hill A, McMahon $\mathrm{H}$, Hall C,et al. Cod liver oil (n-3 fatty acids) as an non-steroidal anti-inflammatory drug sparing agent in rheumatoid arthritis. Rheumatology (Oxford). 2008;47:665-9.

95. 94. Calder PC. n-3 polyunsaturated fatty acids, inflammation, and inflammatory diseases. Am J Clin Nutr 2006;83(6 Suppl):1505S-19S.

96. Arslan F, Keogh B, McGuirk P, Parker AE. TLR2 and TLR4 in ischemia reperfusion injury. Mediators Inflamm 2010;704202.

97. Zeghichi-Hamri S, de Lorgeril M, Salen P, Chibane M, de Leiris J, et al. Protective effect of dietary n-3 polyunsaturated fatty acids on myocardial resistance to ischemia-reperfusion injury in rats. Nutr Res 2010;30:849-57.

98. Pan HC, Kao TK, Ou YC, Yang DY, Yen YJ, Wang $\mathrm{CC}$, et al. Protective effect of docosahexaenoic acid against brain injury in ischemic rats. J Nutr Biochem 2009;20:715-5.

99. Delerive P, Gervois P, Fruchart JC, Staels B. Induction of IkBa expression as a mechanism contributing to the anti-inflammatory activities of peroxisome proliferators-actived receptor a activators. J Biol Chem 2000; 275: 36703-7. 